\title{
Estado y prospectiva de las posibilidades de mitigación de impacto del cambio climático en la región Costa Atlántica*
}

\section{Status and Prospective of Potential Impact Mitigation of Climate Change on the Atlantic Coast Region}

\author{
Reinaldo Guerrero Gutiérrez ${ }^{* *}$ \\ Juan de Dios Flórez Díaz ${ }^{* * *}$ \\ Luz Elena García García ${ }^{* * * *}$
}

Recibido: 13/06/2013

Aprobado Evaluador Interno: 10/11/2013

Aprobado Evaluador Externo: 10/02/2014

\section{Resumen}

La Universidad de Manizales, a través del Centro de Investigaciones en Medio Ambiente y Desarrollo Sostenible (CIMAD) y la Maestría en Desarrollo Sostenible y Medio Ambiente, elabora

\section{Abstract}

The University of Manizales, through the Center for Research on Environment and Development (CIMAD) and the Masters in Sustainable Development and Environment,

doi:10.11144/Javeriana.PAPO19-1.eppm

* Artículo de investigación.

** Reinaldo Guerrero Gutiérrez, Licenciado en Ciencias Naturales, Universidad del Magdalena. Asistente de Investigación, candidato a Magíster en el Programa de Maestría en Desarrollo Sostenible y Medio Ambiente, Línea de Investigación Desarrollo Social y Humano, Facultad de Ciencias Contables, Económicas y Administrativa de la Universidad de Manizales. Correo electrónico: reigu21@hotmail.com

*** Juan de Dios Flórez Díaz, Ingeniero Agrónomo, Universidad del Magdalena. Especialista en Gerencia del Talento Humano. Asistente de Investigación, candidato a Magíster en el Programa de Maestría en Desarrollo Sostenible y Medio ambiente, Línea de investigación Desarrollo Social y Humano, Facultad de Ciencias Contables, Económicas y Administrativas de la Universidad de Manizales. Correo electrónico: jd71florez@gmail.com.

**** Luz Elena García García, Economista, Universidad de Manizales. Magíster en Pedagogías Activas y Desarrollo Humano, alianza Cinde-Universidad de Manizales. Candidata a doctora Programa Conocimiento y Cultura en América Latina, Instituto Pensamiento y Cultura en América Latina (Ipecal, México). Docente, investigadora y directora Línea de Investigación Desarrollo Social y Humano de la Maestría en Desarrollo Sostenible y Medio Ambiente, miembro del Grupo Centro de Investigación en Medio Ambiente y Desarrollo Sostenible (CIMAD) Facultad de Ciencias Contables, Económicas y Administrativas de la Universidad de Manizales. Correo electrónico: luzeg@umanizales.edu.co, luzelenagarcia@me.com 


\section{Reinaldo Guerrero Gutiérrez, Juan de Dios Flórez Díaz y Luz Elena García García}

y ejecuta el proyecto de investigación "Estado y prospectiva de las posibilidades de adaptación y mitigación de impacto del cambio climático en diferentes regiones del país”. Este proyecto pretende, entre otros objetivos, conocer las condiciones mitigantes de los impactos del cambio que puedan estar presentes en las poblaciones vulnerables y su disposición a la prevención, así como describir las acciones de mitigación del riesgo desarrolladas a nivel estatal y privado.

En este artículo se dan a conocer las condiciones de mitigación en la Costa Atlántica Colombiana y las acciones públicas y privadas que se están desarrollando para alcanzar este cometido, entendiéndose mitigación como la intervención humana para reducir los impactos del clima sobre la población afectada por fenómenos climáticos a través de estrategias de reducción de fuentes y emisiones de gases efecto invernadero (GEI) y la promoción de sumideros (conservación y restauración de bosques).

\section{Palabras clave:}

investigación; mitigación; intervención humana; reducción de emisiones; cambio climático; acciones públicas y privadas develops and implements the research project "State and prospects of possibilities for adaptation and mitigation of the impact of climate change in different regions of the country". This project proposes, among others, to explore the conditions mitigating the impacts of change that may be present on vulnerable populations and their willingness to prevention, and also to describe the risk mitigation actions developed at the state and private sectors.

In this article we present the mitigation conditions on the Colombian Atlantic Coast, and the public and private actions that are being developed to achieve this goal, understanding mitigation as human intervention to reduce climate impacts on the affected population by climatic events through source reduction strategies and emissions of Greenhouse Gases (GHG) and promotion of sinks (forest conservation and restoration).

\section{Keywords:}

research; mitigation; human intervention; emissions reduction; climate change; public and private actions 


\section{Introducción}

En la Investigación titulada Estado y prospectiva de las posibilidades de adaptación y mitigación de impacto del cambio climático en diferentes regiones del país se seleccionó la Costa Atlántica como región objeto de estudio, y para ello se realizaron consultas sobre los referentes teóricos más relevantes acerca de la categoría de la mitigación del cambio climático y de las concepciones, políticas, planes, programas, proyectos, acciones y posibilidades en dicha región colombiana.

Este artículo consta de tres partes: en la primera se hace un abordaje teórico de esta categoría como clave para entender las variaciones y tendencias en los últimos años sobre la mitigación de los efectos del cambio climático. En la segunda parte se exponen las políticas, programas, proyectos y acciones de mitigación, así como las posibilidades en la región con base en los análisis e interpretaciones de la información cuantitativa y cualitativa recolectada en las diferentes técnicas de investigación aplicadas, y en la última sección se muestran las conclusiones sobre la categoría de mitigación de acuerdo con los objetivos y los resultados de la interpretación de la información suministrada por la población investigada.

En cuanto a las políticas, se resaltan en especial los lineamientos del Consejo Nacional de Política Económica y Social (Ministerio de Minas y Energía, 2013), donde se muestra la ruta trazada por el Gobierno nacional para enfrentar los retos y amenazas de este fenómeno. Igualmente, se hace referencia a la necesidad de fortalecimiento de las capacidades institucionales para la gestión integral de riesgos en el Caribe Colombiano, específicamente el Nodo Regional Cambio Climático Caribe, iniciativa avalada por el Sistema Nacional de Prevención y Atención de Desastres (SNPAD) y el Programa de las Naciones Unidas para el Desarrollo en Colombia (PNUD).

La presente investigación propone como objetivo general analizar e interpretar las acciones que se vienen realizando en diferentes regiones del país para mitigar los efectos adversos del cambio climático y la capacidad de adaptación de las diversas regiones, con el fin de afrontar las situaciones derivadas de dicho cambio y reducir la vulnerabilidad de la población.

Dentro de los objetivos específicos encontramos los siguientes asociados a la categoría de mitigación: describir las acciones de mitigación del riesgo desarrolladas en el nivel estatal y privado, y conocer las condiciones mitigantes de los impactos del cambio que puedan estar presentes en las poblaciones vulnerables y su disposición a la prevención.

En el desarrollo de este trabajo de investigación, la metodología es de enfoque mixto, empírico-analítico, pues se busca analizar, describir y tipificar acciones de mitigación, y hermenéutica, en tanto pretende comprender las posibilidades de mitigación en la Costa Atlántica Colombiana.

Los instrumentos de recolección de información fueron las encuestas, entrevistas y grupos focales, las cuales se aplicaron a comunidades generales y vulnerables, personas 
vinculadas a entidades u organismos ambientales con conocimientos sobre la problemática ambiental relacionada con el cambio climático.

En el caso de las encuestas, la muestra ascendió a doscientos setenta y ocho (278) personas, distribuidas en los ciento noventa y cuatro (194) municipios de los siete (7) departamentos que conforman la Costa Atlántica (Atlántico, Bolívar, Magdalena, Guajira, Cesar, Córdoba y Sucre). Además, se realizaron veinte (20) entrevistas semiestructuradas, diez (10) grupos focales institucionales y veinte (20) con comunidades. En el archipiélago de San Andrés y Providencia no se aplicaron instrumentos. El estudio se enfocó en la parte continental.

En su gran mayoría, las encuestas se realizaron de manera virtual a funcionarios de los sectores público y privado que tuvieran relación directa con el tema ambiental tales como: Secretarías de Ambiente, Secretarías de Planeación, Secretarías de Agricultura, UMATAMAS, ONGs, funcionarios de nivel medio de Corporaciones Autónomas Regionales (CARs) y demás dependencias del ámbito departamental y municipal que estuvieran relacionadas con los objetivos de la investigación.

En la encuesta se indagó sobre la comunidad en general, los aspectos de mitigación al cambio climático que se están trabajando en la región Costa Atlántica y la efectividad de las acciones para la mitigación del cambio climático, además de la utilización de la matriz Sistema de Información Geográfica (SIG).

La entrevista semiestructurada se realizó a representantes o autoridades departamentales y regionales que conocen a profundidad el tema estudiado, como directores o subdirectores de corporación, secretarios de medio ambiente, directores de grupos de investigación que han abordado el tema, directores o subdirectores del Instituto de Hidrología Meteorología y Estudios Ambientales (IDEAM), nodos regionales de Cambio Climático, directores de ecoregiones, entre otros, a quienes también se les preguntó por la categoría mitigación.

A través de esta técnica, se buscó información sobre las políticas para mitigar el cambio climático, programas y proyectos de mitigación, la articulación de éstas con la planeación y actuación de autoridades ambientales, los logros y la proyección en materia de mitigación al cambio climático.

Mediante los grupos focales institucionales, se indagó la percepción de los funcionarios ambientales con relación a la mitigación en la ejecución de proyectos importantes en la región orientados a disminuir las emisiones de gases de efecto invernadero (GEI) y/o a capturar carbono. También se cuestionaron las proyecciones futuras sobre mitigación, apoyadas en una actuación sistemática y coordinada de la clase dirigente en la región para afrontar el cambio climático en las diferentes provincias.

Se utilizó la misma técnica de recolección de información aplicada a comunidades vulnerables frente a los efectos ambientales del cambio climático. En estas poblaciones se 
indagó sobre las políticas claras para mitigar el cambio climático, programas y proyectos enfocados a disminuir la generación de GEI o a la captación de $\mathrm{CO}_{2}$. Igualmente, se preguntó por los logros en materia de mitigación, la prospectiva en la articulación de políticas ambientales, el compromiso de las autoridades y el funcionamiento del Comité de Atención de Desastres en cuanto el componente de mitigación.

\section{Análisis e interpretación sobre la categoría mitigación del cambio climático}

La mitigación comprende las intervenciones humanas, a través de la aplicación de políticas, acciones y estrategias de reducción de emisiones de fuentes y emisiones de GEI y la promoción de sumideros naturales (conservación y restauración de bosques) que contribuyan a reducir los impactos del clima sobre la población afectada por fenómenos climáticos.

La mitigación se enmarca dentro del Mecanismo de Desarrollo Limpio, el cual representa una posibilidad para los países en desarrollo, entre ellos Colombia, en el sentido de i) generar reducciones de emisiones verificadas de manera competitiva, crear nuevas oportunidades de negocios, atraer inversión extranjera y generar fuentes de divisas; ii) generar beneficios adicionales, como difusión de tecnologías ambientalmente sanas, mejoramiento de la calidad del medio ambiente local, generación de empleo; y iii) fortalecer las instituciones y los sectores en relación con la mitigación del cambio climático. Es necesario el mercadeo de los proyectos en el escenario internacional a través de los diferentes gobiernos, fondos y empresas en países desarrollados interesados en adquirir estas reducciones de emisiones (Ministerio del Medio Ambiente, 2000; Ministerio del Medio Ambiente e IDEAM, 2001).

La estrategia abarca cuatro aspectos: i) definición de la política de venta de servicios ambientales de mitigación de cambio climático, ii) consolidación de una oferta de reducciones demisiones verificadas, iii) mercadeo internacional de la oferta de reducciones de emisiones verificadas, y iv) coordinación, seguimiento y evaluación de la estrategia.

Dada la alta prioridad del tema de cambio climático y sus posibles impactos ambientales, sociales y económicos asociados, el Gobierno colombiano ha desarrollado las siguientes acciones: i) Estudio de Estrategia Nacional para la Implementación del Mecanismo de Desarrollo Limpio (MDL) en Colombia; ii) Primera Comunicación Nacional ante la Convención Marco de las Naciones Unidas sobre Cambio Climático; iii) Oficina Colombiana para la Mitigación del Cambio Climático (OCMCC); iv) Lineamientos de Política de Cambio Climático; v) Incentivos tributarios para proyectos de venta de reducciones de emisiones verificadas; vi) Firma de memorandos de entendimiento; y vii) Identificación de potencial.

Aunque Colombia no tiene compromisos con los países de las Naciones Unidas para reducir las emisiones de GEI y no aporta una cantidad determinante a diferencia de otros países (0,37\% de las emisiones globales para el año 2004), sí ha estado inserta en la 
dinámica que busca atender el cambio climático mediante el establecimiento de diferentes políticas que promueven el desarrollo sostenible asociado a bajas emisiones de gases, reflejando así una evolución en materia de mitigación a nivel nacional e internacional.

En el nivel internacional, siguiendo los mecanismos de flexibilidad establecidos en el Protocolo de Kioto, hasta el momento se han registrado en Colombia cerca de cincuenta (50) transacciones, de las cuales treinta y ocho (38) corresponden a proyectos del Mecanismo de Desarrollo Limpio y doce (12) a Implementación Conjunta. El volumen total de reducciones de emisiones es de aproximadamente ciento siete (107) millones de toneladas de dióxido de carbono equivalente, transadas a un precio estimado entre 3 y 4 dólares por tonelada. Los proyectos más frecuentes son los de conversión de energía hidráulica (26\%), eólica (23\%), biomasa (23\%); los proyectos de captura de metano en rellenos sanitarios (13\%) y, en menor proporción, los proyectos de eficiencia energética, sustitución de combustibles, geotermia y cogeneración. A la fecha sólo se ha transado un proyecto forestal bajo el esquema de Implementación Conjunta.

En el nivel nacional se resaltan, entre otros, los instrumentos de gestión ambiental y financieros que incentivan la adquisición de tecnologías y equipos que contribuyan a la reducción de emisiones de GEI. El país, bajo la Ley 788 de 2002, estableció incentivos de exención de renta por quince (15) años a la venta de energía producida a partir de fuentes renovables como la eólica, biomasa o residuos agrícolas (Artículo 18 ley 788/2002). De tal gestión se pueden beneficiar las empresas generadoras, siempre y cuando se obtengan y se vendan certificados de reducción de GEI, y se destine el 50\% de los recursos obtenidos por este concepto a obras de beneficio social. De otro lado, el Artículo 95 establece que la importación de maquinaria y equipos destinados a proyectos que generen certificados de reducción de GEI, estará exenta del impuesto al valor agregado (IVA) del producto y la prestación de servicios.

Dentro del marco de la legislación colombiana, se estableció el reglamento técnico de eficiencia energética, el cual contempla dentro de sus objetivos establecer medidas para fomentar el uso racional de la energía, mitigar los impactos medioambientales generados por la utilización de maquinaria, fomentar equipos de uso final de alta eficiencia energética y proteger al consumidor.

Para el caso de Colombia, el país cuenta con tres grandes cualidades que podrían permitirle ser un importante actor internacional. Primero que todo, su cobertura boscosa relativamente bien conservada. En segundo lugar, la matriz energética que en un $65 \%$ ya es limpia, el país utiliza la energía hidroeléctrica para generar gran parte de los 13.500 megavatios $(\mathrm{Mw})$ instalados en su sistema interconectado. Por último, el sector empresarial Colombiano ha avanzado, a través de procesos de mejoramiento, en el uso eficiente de la energía.

De otra parte, el uso de combustibles fósiles ha generado niveles preocupantes de contaminación por el incremento de la cantidad de dióxido de carbono en la atmósfera 
que produce el denominado calentamiento global; por esto se han implementado medidas conducentes a disminuir la producción de los gases de efecto invernadero. Mecanismos como los bonos de carbono son herramientas internacionales útiles buscando tal fin. Colombia, así como la mayoría de países, ha implementado políticas públicas basadas en el beneficio social y económico buscando modificar comportamientos ambientales de los actores que intervienen en la transformación y uso de la energía mediante incentivos que combinan políticas de contaminación con regulación de emisiones.

En la presente investigación, el estudio de esta categoría comprende un análisis sobre las políticas, los planes, los proyectos y los programas generados en la región de la Costa Atlántica, así como las percepciones, los logros y las posibilidades en materia de mitigación.

\section{Políticas asociadas a la mitigación del cambio climático en Colombia}

En Colombia, las políticas de mitigación se rigen por los documentos del Consejo Nacional de Política Económica y Social (CONPES), organismo técnico asesor del ejecutivo y máxima autoridad nacional de planeación estratégica en los campos social y económico.

Los lineamientos de Política de Cambio Climático, aprobados por el Consejo Nacional Ambiental en Julio 16 de 2002, contemplan las estrategias de promoción de actividades de reducción de emisiones de GEI dentro del esquema del Mecanismo de Desarrollo Limpio (MDL).

El fenómeno del Cambio Climático es tomado en cuenta como factor perturbador del presente y el futuro económico y social del país; por lo tanto, en el documento CONPES 3242, "Estrategia institucional para la venta de servicios ambientales de mitigación del cambio climático", se enmarca en el Plan Nacional de Desarrollo 2003- 2006, en relación con los programas de la sostenibilidad ambiental, la generación de ingresos y empleo verde. En estos programas se contempla el impulso de ocho (8) proyectos de venta de servicios ambientales de mitigación del cambio climático.

Cabe resaltar que el MAVDT crea el Grupo de Mitigación de Cambio Climático (GMCC) en el 2005 con el objetivo de promover el desarrollo de proyectos MDL de alta calidad en el país. Hasta diciembre de 2009, las actividades desarrolladas por el GMCC han permitido la consolidación de un portafolio nacional de ciento cuarenta y cuatro (144) proyectos, de los cuales cuarenta y nueve (49) tienen aprobación nacional por solicitud directa de los proponentes, veinte (20) proyectos están registrados ante la CMNUCC y seis (6) cuentan con Certificado de Reducción de Emisiones (CER).

La distribución de estos proyectos por sectores se resume así: energético (31,25\%), transporte $(8,3 \%)$, forestal $(11,8 \%)$, industrial $(31,25 \%)$ y residuos $(17,36 \%)$. El potencial anual de reducción de emisiones de GEI del total de los proyectos MDL que hacen parte del portafolio nacional es de aproximadamente 16.402.496 toneladas 
de $\mathrm{CO}_{2}$ equivalente, reducciones que podrían generar potenciales ingresos al país de unos USD \$ 152.000.000.

En Septiembre de 2009, Colombia se convierte en el quinto país de Latinoamérica y décimo segundo del mundo en proyectos elegibles al Mecanismo de Desarrollo Limpio del Protocolo de Kioto, luego de que el Relleno Sanitario Doña Juana de Bogotá (Ingresos estimados USD \$ 9.000.000) y la Planta de Tratamiento de Aguas Residuales Cañaveralejo de Cali obtuvieran el respectivo registro de las Naciones Unidas; estos contribuyen con la reducción de más de 827.384 toneladas anuales de $\mathrm{CO}_{2}$. De esta manera, el país completa veinte (20) proyectos registrados, con lo cual supera a países como Israel, Sudáfrica, Argentina, Ecuador, Costa Rica y Panamá.

Específicamente en la Costa Atlántica, el Nodo Regional de Cambio Climático Caribe e Insular fue creado en con el objeto de desarrollar actividades técnicas como apoyo para la realización de un diagnóstico en las situaciones críticas de la jurisdicción con relación al cambio climático y proponer acciones para su adaptación y mitigación. Busca promover y apoyar la inclusión y desarrollo de acciones que contribuyan a reducir los riesgos asociados al cambio climático, así como logar su adecuada inclusión en los diferentes instrumentos de planificación territorial.

Con la conformación del Nodo Regional también se busca dar cumplimiento a lo establecido en la Convención Marco de las Naciones Unidas sobre cambio climático y en el Protocolo de Kioto, los cuales tienen por objetivo reducir las emisiones de gases que causan el calentamiento global.

Entre los objetivos principales del Nodo Caribe se encuentran promover e impulsar el eje temático de Cambio Climático; entre otros (Nodos Regionales, s.f.).

\section{Políticas de mitigación en la Costa Atlántica}

Las CARs, en apoyo a la iniciativa que busca poner en marcha políticas y prácticas que contribuyan a la adaptación y mitigación de los efectos del cambio climático en la Costa Caribe, suscribieron un convenio con el Ministerio de Medio Ambiente y Desarrollo Sostenible, el Ministerio del Interior y de Justicia y las autoridades ambientales municipales de toda la región caribe en la ciudad de Riohacha.

Asimismo, con el objetivo de generar una dinámica regional de mitigación y adaptación al cambio climático en el Caribe, se conformó el Nodo Regional de Cambio Climático Caribe e Insular.

Cuando en la presente investigación se pregunta a los funcionarios y comunidades en general por la existencia de una política clara de mitigación de los efectos del cambio climático para disminuir la generación de GEI o a la captación de $\mathrm{CO}_{2}$, se encuentra que esta política es desconocida; sólo se ha socializado lo que pudiera alcanzarse sobre los acuerdos del Protocolo de Kioto relacionados con este tema. 
En el momento en que se ausculta a los entrevistados sobre la existencia en la región de una política clara que permita mitigar el cambio climático, la articulación entre las políticas y la actuación de las autoridades ambientales frente a la mitigación del cambio climático, encontramos que son situaciones que se muestran como uno de los problemas de la región Costa Atlántica. Los actores hacen explícita la falta de claridad y de articulación sobre las políticas públicas y el documento CONPES - relacionado con el cambio climático-, y entre el gobierno nacional, departamental y municipal. Así lo expresan textualmente: "Pienso que es importante que este tema de la articulación se le dé prioridad, sobre todo en esta región, porque es una de las regiones donde más ha causado daño el cambio climático”. (Informante clave, Corporación Autónoma del Sur de Bolívar, CSB, 2013).

En este mismo orden de ideas, encontramos estas opiniones: "Bueno, ahora tenemos un convenio con el Ministerio del Medio Ambiente, para incentivar el tema de captura de $\mathrm{CO}_{2}$, pero concretamente una política estructurada en el tema no hay". (Informante clave, Corporación Autónoma Regional del Cesar, 2013); ““'En relación con el cambio climático, digamos que ha faltado mayor articulación entre el gobierno y los municipios y las alcaldías y las gobernaciones y en estos momentos no vemos algo muy concreto sobre en qué se está trabajando unido para mitigar esos efectos del cambio climático". (Corporación Autónoma Regional del Magdalena, 2013)

Los anteriores testimonios confirman la falta de articulación entre políticas y los diferentes entes estatales (Nación, departamentos, municipios), la necesidad de aunar esfuerzos que permitan trabajar conjuntamente en la mitigación del cambio climático y reconocen la mitigación como un asunto prioritario.

No obstante, desde el Gobierno central sí se emiten unas políticas claras sobre la mitigación del cambio climático; en 2002 se crea (OCMCC) que funciona desde el Ministerio de Medio Ambiente y Desarrollo Sostenible. El inconveniente radica en que no hay coordinación entre estas políticas ni en su aplicación en los diferentes entes descentralizados, además del desconocimiento de las comunidades de lo que en materia de mitigación pueden realizar entidades gubernamentales y no gubernamentales en las localidades.

Los representantes de los diferentes entes ambientales demandan una mayor actuación sistemática entre las políticas del Gobierno central acordes con las necesidades intrínsecas de cada región y las diferentes localidades. Además, resaltan la necesidad de inversión de recursos financieros y humanos con conocimiento suficientes para la realización de programas y proyectos de mitigación.

\section{Planes de carácter nacional y regional asociados con la mitigación}

En Colombia, algunos de los planes que tienen en cuenta la mitigación del cambio climático son el Plan Nacional de Desarrollo (PND) 2002-2006 "Hacia un Estado comunitario"; la estrategia institucional para la venta de servicios ambientales derivados de la mitigación 
de cambio climático (Ministerio de Minas y Energía, 2013); el (PND) 2006-2010 “Estado comunitario: desarrollo para todos"; Visión Colombia II Centenario 2019; y los acuerdos de cooperación multilateral, principalmente relacionados con proyectos MDL, entre otros.

La estrategia propuesta en el documento CONPES (Ministerio de Minas y Energía, 2013) busca promover la incursión competitiva de Colombia en el mercado internacional de reducciones verificadas de emisiones de GEI. Para ello establece un marco institucional para la definición de la política de venta de este servicio ambiental. El servicio ambiental de mitigación está relacionado con la venta de reducciones verificadas de GEI. El mercado de reducciones está basado en acuerdos de la comunidad internacional para hacer frente al cambio climático, que constituye el problema ambiental global más importante de este siglo.

Entre los planes y estrategias sectoriales en Colombia, se han identificado algunos sectores que desarrollan acciones frente al tema de mitigación del cambio climático.

El sector energético cuenta con diferentes planes y programas que potencialmente contribuyen con la reducción de emisiones GEI como por ejemplo: el Plan Energético Nacional del Ministerio de Minas y Energía (MME) y el Plan Energético Nacional, 20062025 programa URE (Uso Racional de Energía) y FNCE (Fuentes No Convencionales de Energía) (Ministerio de Minas y Energía, 2001); programa PROURE (Uso Racional y Eficiente de Energía y otras formas de Energía no Convencionales) (Ministerio de Minas y Energía 2001 y 2003); subprogramas de Zonas no Interconectadas; y Programa Metano al Mercado con Environmental Protection Agency, USA, y MAVDT.

El sector transporte tiene actualmente en operación o construcción ocho (8) Sistemas Integrados de Transporte Masivo (SITM) en las principales ciudades del país: Bogotá D.C., Soacha, Barranquilla, Bucaramanga, Santiago de Cali, Cartagena, Medellín (Valle de Aburrá) y Pereira (Dos Quebradas). Se espera que estos SITM en el futuro tengan considerables efectos en la reducción de emisiones de dióxido de carbono (CO2). En promedio, para estos proyectos se estima un potencial de reducción de emisiones de GEI anual cercano a 810.726 toneladas de $\mathrm{CO}_{2}$.

En el sector industrial, según la encuesta de opinión industrial conjunta realizada por la Asociación Nacional de Empresarios de Colombia (ANDI) (2009), dentro de las medidas que tomarán los empresarios en los próximos cinco años para mitigar el cambio climático se destacan principalmente la eficiencia energética $(80,3 \%)$ y la educación y sensibilización del personal de sus empresas (78,4\%). Estas respuestas combinan, de manera prometedora, medidas tecnológicas con acciones educativas. Por otra parte, un 47,4\% efectuarán cambios en sus procesos productivos y un 29,1\% en sus productos; mientras tanto, un $38 \%$ proyecta extender las exigencias a su cadena de suministro, 24,9\% aportará para la protección de los ecosistemas y 24,4\% planea tomar acciones concretas para neutralizar las emisiones de carbono. 
En el desarrollo de las medidas de mitigación pertinentes al módulo "uso del suelo, cambio de uso y silvicultura" (Sucus) se analiza el sector forestal desde el ámbito de los diferentes instrumentos de política que han diseñado medidas de mitigación; tal es el caso de la Política de Bosques (DNP, 1996), el Plan Verde (DNP \& MMA, 1998) y el Plan Nacional de Desarrollo Forestal (2000).

El proyecto "Emisiones por Deforestación en Colombia" del 2009, avanza en el desarrollo de los protocolos nacionales y nacionales de procesamiento de imágenes para monitorear la deforestación, la estimación de carbono en bosques y otras coberturas vegetales, y el monitoreo de biomasa por medio de sensores remotos.

Igualmente, se ha llevado a cabo un ejercicio preliminar para la cuantificación de la tasa de deforestación para el periodo 2000-2007. Se espera generar la línea base histórica de deforestación en el país, la estimación de los stocks de carbono para los diferentes tipos de ecosistemas boscosos, la elaboración del mapa de áreas potenciales para la implementación de proyectos REDD en Colombia, y también desarrollar una plataforma web institucional para el apoyo a los desarrolladores de estos proyectos.

En el sector agrícola, la gestión ambiental se establece con dos instrumentos de planificación que integran algunas medidas relacionadas con la mitigación del cambio climático. El primero es la Agenda Ambiental Interministerial entre el MAVDT y el Ministerio de Agricultura y Desarrollo Rural (MADR), y el segundo, el Plan Estratégico Ambiental del Sector Agropecuario (PEASA). Ambos definen algunas líneas de acción que integran medidas de mitigación del cambio climático. También se cuenta con algunos proyectos en el tema de mitigación dentro de la estrategia de investigación que creó el MADR llamado "Agricultura y Cambio Climático”. Al respecto existen algunas líneas de investigación como Agricultura y cambio climático con la alianza IDEAM - Corpoica, y Ganadería y sistemas silvopastoriles con apoyo de El Fondo Mundial Para el Medio Ambiente (GEF) y el Banco Mundial.

En el sector residuos, varias políticas en Colombia han contribuido a la mitigación del cambio climático. Entre ellas, en el PND 2002-2006 se establece el programa de Sostenibilidad Ambiental con el fin de fomentar el mercado de empresas dedicadas al aprovechamiento de residuos sólidos e impulsar iniciativas sectoriales para el desarrollo de proyectos de reducción de emisiones y aprovechamiento de metano en rellenos sanitarios. Asimismo, se destaca el avance del MAVDT frente al objetivo de gestionar, prestar asistencia técnica e impulsar la formulación y desarrollo de los Planes de Gestión Integral de Residuos Sólidos (PGIRS), que para el cuatrienio 2002-2006 se cumplió en 532 municipios de Colombia de los cuales ciento cuarenta y dos (142) corresponden a la Costa Atlántica (SUI, 2008)

\section{Proyectos de mitigación}

Respecto al tema de los proyectos orientados a disminuir las emisiones de gases y efectos invernadero y/o a captura de carbono, el desarrollo y medidas de mitigación 
en cuanto a emisiones de gases de efecto invernadero es responsabilidad delas autoridades ambientales departamentales como las CARs. Éstas desarrollan la aplicación de decretos que se hacen a nivel nacional por el Ministerio de Medio Ambiente y Desarrollo Sostenible, las CARs le hacen seguimiento al cumplimiento de los decretos en cuanto a la emisión de gases de fuentes fijas y móviles que son las que están generando en este momento este tipo de emisiones.

En este aspecto se hace un seguimiento a través de resoluciones: los permisos de emisiones atmosféricas que se otorgan para este tipo de emisiones establecen que se deben monitorear periódicamente. Como las empresas deben cumplir esas normas, estos procesos son monitoreados pero no hay medidas correctivas para la mitigación.

En los departamentos que tienen explotación carbonífera y su transporte, se tiene una red de monitoreo sin impacto y sin seguimiento y, algunos equipos de estos están ubicado en zonas inadecuadas que no permiten una medición real de la situación (demasiada arborización) y se generan partículas de carbón o contaminantes atmosféricos. Por esto es necesario articular con las CARs de los diferentes departamentos costeros, las autoridades civiles y las comunidades afectadas para coordinar la relación que tienen con la contaminación atmosférica y los gases que se pueden generar en las principales ciudades como Riohacha, Maicao, Valledupar, Aguachica, Santa Marta, Barranquilla, Sincelejo y Montería. No obstante, hoy no se tiene posibilidad de medición de las emisiones de gases de efecto invernadero, sólo las redes de monitoreo que tienen algunos departamentos.

Con base en los resultados de la presente investigación en la Región Costa Atlántica, a continuación se muestran algunas percepciones que tienen los encuestados y comunidades en general sobre los proyectos de mitigación tendientes a la captura de carbono, procesos de reforestación y capacitación.

Sobre los proyectos de mitigación, los entrevistados expresan:

En el departamento de la Guajira adelantamos un estudio, donde hicimos un proyecto de captura de carbono en un sitio por acá en Mingeo sobre captura de carbono en el suelo, sobre las reforestaciones que ha venido haciendo la corporación a través de su existencia, porque es que la reforestación no busca solamente proteger las cuencas y el recurso hídrico; también tenemos allí un agro proyecto de captura de carbono, que lo estamos adelantando, también tenemos eso en nuestro plan de acción 2012-2015. (Guajira, 2013)

En el departamento de Sucre dicen: "La región está siendo beneficiada con proyectos desde nivel central y principalmente ejecutados por CARSUCRE, como reforestaciones, protección de cuencas y cuidados del ambiente". (Informante clave: Secretaria de Gobierno, Colosó-Sucre Municipio de Colosó Sucre, 2013), y: 
Bueno precisamente el departamento del César viene realizando el Plan Departamental de Gestión del Riesgo, hay dentro de sus planes de acción tiene la reducción de riesgos esto ayuda a reducir los efectos que se puede generar para un cambio climático; entonces, si hay unas proyecciones aspiramos gestionar los recursos propios pertinentes para poder avanzar con mayor celeridad y oportunidad en el tema de la mitigación de los riesgos en el departamento del Cesar. (Informante clave: Dirección Gestión del Riesgo D. d., 2013)

En el departamento de Córdoba:

Aunque es de conocimiento general que Colombia pertenece al grupo de países en vía de desarrollo no incluido en la convención marco de las naciones unidas para el CC, los cuales no cuentan con una meta concreta y obligatoria de reducción de gases de efecto invernadero (GEI), y se les permite incluso un aumento de sus emisiones con el fin de avanzar en la realización de sus modelos de desarrollo y sociales, Pero estamos para eso, debemos trabajar para mejorar esta situación, iniciando en primer lugar por nuestra región. Es por esto que nace la Red de Cambio Climático y Seguridad Alimentaria (RICCLISA) nodo Córdoba. Aquí en la región se cuenta con aquellas áreas reforestadas, que inicialmente no fueron cultivadas con este fin, pero actualmente contribuyen mucho en este campo. (Informante clave: Director RICCLISA, 2012)

En el departamento del Magdalena se encontró que:

Se adelantan proyectos de MDL, en coordinación con la Corporación Autónoma Regional del Magdalena, y el Programa de Reforestación Comercial se desarrolló en el Núcleo del Magdalena Bajo Seco, en los municipios ribereños de Piñón, Plato, Santa Bárbara de Pinto, Tenerife y Zapayán en el departamento de Magdalena, y en los municipios de La Gloria y Tamalame que en Cesar con el cofinanciamiento de la Corporación Autónoma Regional del Río Grande de la Magdalena (CORMAGDALENA), el Fondo para el Financiamiento del Sector Agropecuario (FINAGRO) y la asistencia técnica y la operación por parte de la Oficina Nacional Forestal de Francia, (ONF Andina). (CORPAMAG I. c., 2013)

En el departamento de Bolívar:

¿La política realmente digamos que a nivel nacional tú sabes que se viene trabajando en esto, tanto por parte del Ministerio del Medio Ambiente como de otras entidades como el IDEAM, sobre el tema del cambio climático y la situación de acá en esta zona todavía digamos que no está bien estructurada, todavía se necesita hacer más análisis, más estudio y obviamente hay que seguir trabajando sobre este tema, no podemos todavía determinar 
que se estén generando unos programas o proyectos que verdad puedan contribuir con este tema. (Informante clave vinculado a la Corporación Autónoma del Sur de Bolívar, 2013).

Como puede observarse en los testimonios que devienen de los entrevistados, se reconoce la existencia de programas y proyectos enfocados a la captura de carbono y procesos de reforestación para la conservación del recurso hídrico, la protección ambiental y la gestión del riesgo.

\section{Educación ambiental}

$\mathrm{El}$ escenario anterior es diferente en la encuesta en donde se indaga a la comunidad en general sobre la existencia de programas y proyectos de mitigación. Se encuentra que un alto porcentaje (48.9\%) de los encuestados considera a la educación ambiental como el aspecto de mitigación que más se viene trabajando en la región, seguido de la realización de proyectos de reforestación y educación ambiental (39.9\%), es decir que el 88.8\% del total de los encuestados reconoce a los proyectos de educación ambiental como claves para la mitigación. Sólo un $6.5 \%$ alude a los proyectos de reforestación y un $4.0 \%$ considera que no se está trabajando en proyectos de mitigación. En menor proporción, se refieren a sólo generación y uso de energías alternativas (0.4\%), generación y uso de energías alternativas y proyectos de reforestación (0.4\%).

Teniendo en cuenta la importancia de los procesos de formación y capacitación de diversos sectores de la población en materia de la mitigación, la construcción de una cultura de la prevención del Caribe Colombiano se convierte en un imperativo. Los gobernantes de turno deben comprometerse a involucrar en sus planes de desarrollo y de ordenamiento territorial el componente de la gestión del riesgo, tema de vital interés que demanda una gran responsabilidad por parte del sector gubernamental y el sector privado; este último ya ha empezado a adoptar programas de mitigación que sean pertinentes ante la crisis ambiental.

Los actores consultados a través de las diferentes técnicas afirman que en la región se han tenido logros importantes de mitigación frente al cambio climático, sobre todo en relación con la educación ambiental en el caso de la comunidad en general, y proyectos de captura de carbono y reforestación según la percepción de los funcionarios públicos y las autoridades ambientales. Además, una subcategoría clave para tener en cuenta en la mitigación frente al cambio climático es la gestión del riesgo.

\section{Gestión del riesgo}

A partir de la sanción de Ley 1523 del 2012, el Gobierno colombiano, a través de la Unidad Nacional de Gestión del Riesgo, está tratando de coordinar la actuación de la Nación, del departamento y los municipios, a través de la elaboración y la implementación de planes 
departamentales y municipales de gestión del riesgo. Estos planes van a garantizar una planeación previa a la ocurrencia de los eventos y tener unas medidas de mitigación y de atención de emergencias, para así estar organizados en cuanto al tema del manejo de los desastres, saber qué hacer ante una emergencia, disminuir la improvisación e implementar acciones que permitan reducir los riesgos existentes y latentes que en cualquier momento puedan ocurrir en la región.

De igual manera, los entes territoriales deben formular, al tenor de la Ley 1523 de 2012, el Plan Ambiental del Riesgo con la participación de todas las organizaciones sociales, culturales y ambientales del municipio y departamento. La comunidad educativa, con la orientación de la Dirección de Gestión del Riesgo, tiene la misión de formular e implementar los planes escolares para la gestión del riesgo con el propósito de preparar al recurso humano para que dé una respuesta oportuna y efectiva en caso de desastres y emergencias.

En Ciénaga existe la Fundación para la Prevención y Reducción del Riesgo en Colombia (FUNPRAECOL), organización conformada por treinta y cinco (35) promotores comunitarios en riesgo, que ha asumido el compromiso de divulgar y concienciar a la ciudadanía de Ciénaga en todo este proceso de la cultura ambiental y el riesgo. Se requiere también del empoderamiento de la sociedad civil en el manejo de estos asuntos y, fundamentalmente, de un mayor compromiso de gobernantes más sensibles, aptos y capacitados en todo lo inherente a las crisis ambientales que sean capaces de asumir liderazgo y que den respuestas efectivas ante cualquier amenaza que genere algún tipo de riesgo a la región. (Informante clave: Director Departamental de Gestión del Riesgo, 2013)

En relación con lo anterior, a través de la técnica grupo focal a expertos ambientales, un informante clave manifiesta:

Ya se habla de construir una cultura si se empieza ya a construir una cultura de la gestión del riesgo en la región. De hecho, desde el año 2009, con el apoyo de la empresa Ecopetrol y el Secretariado de Pastoral Social Caritas Atlántico, se inició un proyecto de organización de promotores en gestión del riesgo como estrategia para la instalación de los Sistemas de Alertas Tempranas (SAT) en los departamentos de Atlántico, Bolívar, Cesar y Magdalena, proceso que culmina en el 2012 con un Diplomado en Gestión Ambiental del Riesgo. Producto de ello es la conformación de un número significativo de Asociaciones y Fundaciones comprometidas en desarrollar acciones encaminadas a enfrentar y mitigar los posibles impactos que pueda generar el cambio climático en la región. (Informante clave: Dirección Departamental de Gestión del Riesgo, 2013) 
Se observa que a nivel regional comienzan a formarse grupos con conocimiento en gestión del riesgo en las comunidades afectadas, dinámica que cuenta con la participación estatal y privada y el acompañamiento de actores ambientales, lo que fortalece las posibilidades de contribución en conocimiento social de los lugareños en la mitigación del impacto por el cambio climático.

\section{Acciones sobre mitigación}

En la presente investigación se determinaron las acciones que se vienen emprendiendo en la Región Costa Atlántica a través de diferentes aspectos de mitigación al cambio climático que se están trabajando y la efectividad de las acciones.

En la gráfica 1 se pueden observar los aspectos de mitigación al cambio climático que se vienen trabajando en la Región.

\section{Gráfica.1. Aspectos de mitigación al cambio climático que se vienen trabajando en la región Costa Atlántica}

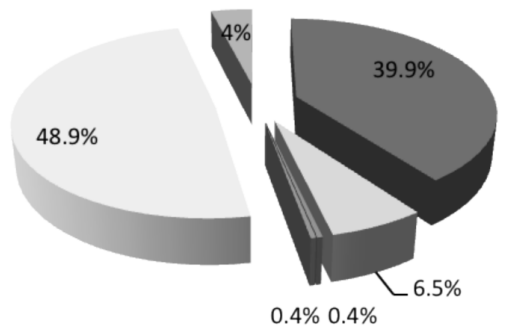

\footnotetext{
Proyectos de reforestación y educación ambiental

Solo proyectos de reforestación

- Solo generación y usos de energías alternativas

- Generación y usos de energias alternativas y proyectos de reforestación

- Solo educación ambiental

No está trabajando proyecto de mitigación
}

Fuente: Elaboración propia del Grupo investigación CIMAD - Región Costa Atlántica

En la Costa Atlántica existen nueve (9) corporaciones autónomas trabajando el tema de educación ambiental y proyectos de reforestación sobre todo en cuencas y micro cuencas. Lo que se busca a través de las CAR'S es formar a la comunidad para que contribuya a identificar y crear las condiciones que coayuden a la construcción del desarrollo integral de los municipios de sus respectivas jurisdicciones e impulsen proyectos en materia ambiental y de desarrollo humano sostenible, que desde una visión integral de la gestión, generan dinámicas que permiten cambios de actitud y comportamiento de la comunidad hacia su entorno e incidiendo en el mejoramiento de la calidad de vida. 
Referente a estos resultados, se observa en la región el cumplimiento de la legislación por parte de las CARs', responsables de asesorar a las entidades territoriales en la formulación de los planes de educación ambiental formal y de ejecutar programas de educación ambiental no formal conforme a las directrices de la política nacional.

En la región se está trabajando más la educación ambiental porque se busca con ella un cambio de actitud en las comunidades, desde el nivel de básica primaria, secundaria y media, de manera articulada y coordinada con los lineamientos establecidos en el Ministerio de Educación y el Ministerio de Ambiente y Desarrollo Sostenible. Se trabaja en los diferentes entes con entidades como Secretarías Ambientales, corporaciones autónomas y las UMATAMAS. Además de esto, también se realizan acciones como la reforestación y labores sociales por parte de algunos estudiantes bajo la supervisión de docentes, en su gran mayoría del área de Ciencias Naturales y Educación Ambiental.

A pesar de los avances de las entidades mencionadas anteriormente con respecto a este tema, la Educación Ambiental, a nivel de la educación básica, media y universitaria, se ha desarrollado más como una actividad coyuntural que como un programa de amplio cubrimiento y cobertura nacional. Esto hace que se inicien procesos de educación ambiental y de reforestación que luego son abandonados por falta de recursos e interés. En cuanto a los proyectos a gran escala de reforestación y de generación de energía alternativa, hace falta más proyección en la Costa Atlántica porque aún no son autosostenibles o viables en toda la región.

Se asume que el tema del cambio climático en la educación es clave para generar conciencia y responsabilidad. Por eso el país requiere generar estrategias de formación, apropiación y comprensión del cambio climático, y que desde las instituciones se brinden directrices gubernamentales y no gubernamentales para emprender conjuntamente acciones respecto al tema.

Es necesario incluir en la educación formal de los grados primarios hasta los niveles superiores los elementos comprensivos de los impactos del cambio climático y las alternativas para afrontarlos como mecanismos para dar inicio a una transformación cultural que permita asumir esta nueva realidad.

En relación con los proyectos de reforestación a gran escala, hay que destacar el proyecto de la firma Pizano S.A., que se vio abocada a establecer su propia reserva forestal con el fin de abastecer sus plantas madereras. Dicha organización cuenta ahora con un cultivo forestal ubicado en el municipio de Zambrano, departamento de Bolívar: Monterrey Forestal, que sobrepasa las 11.000 hectáreas de bosque plantado.

Igualmente, la empresa Refocosta (Reforestadora de la Costa S.A.S.) fue constituida en 1980, empresa de capital privado hoy perteneciente al Grupo Empresarial Valoren, debido a la consciencia de la necesidad de un suministro sostenible de materia prima para el mercado de madera sólida. Desde un principio, Refocosta se ha comprometido a desarrollar 
actividades en el campo de la investigación de especies forestales maderables y a fomentar la reforestación para la comercialización de la madera sólida.

En la generación y uso de energías alternativas, Colombia cuenta desde el año 2004 con un único parque eólico, Jepírachi, instalado cerca del Cabo de La Vela en el departamento la Guajira. Empresas Públicas de Medellín (EPM) puso en funcionamiento este parque eólico con quince (15) aerogeneradores que aportan 19.5 megavatios al Sistema Interconectado Nacional. Jepírachi significa vientos del nordeste (vientos que vienen del nordeste en dirección del Cabo de la Vela) en Wayuunaiki, la lengua nativa Wayuu.

Jepírachi está registrado como MDL por la Convención Marco de las Naciones Unidas para el Cambio Climático. Fue uno de los primeros proyectos MDL firmados en el país y uno de los primeros en todo el mundo, lo cual se constituye en un hito importante en la historia del mercado del carbono. Asimismo, las metodologías de estimación de emisiones y sobre todo las de concertación del proyecto con la comunidad Wayuu, fueron consideradas como modelos por el Banco Mundial y como referencias obligatorias para el desarrollo de proyectos similares en otras partes del mundo.

A raíz de esta experiencia, el Banco Mundial creó el Fondo Comunitario del Carbono (Community Carbon Fund, CCF) con el fin de apoyar proyectos de MDL en comunidades menos favorecidas. Su gestión social se basa en el respeto a la integridad étnica y cultural de la comunidad Wayuu, el establecimiento de relaciones de confianza y la búsqueda de la equidad y el beneficio mutuo.

Para avanzar en el compromiso del Gobierno Nacional con la mitigación de la problemática que enfrenta el país a causa de los efectos del cambio climático, especialmente en regiones afectadas por inundaciones fuertes o por sequias prolongadas, el MADS puso en marcha el proyecto Reducción del Riesgo y la Vulnerabilidad Frente al Cambio Climático en la Depresión Momposina en Colombia.

Se evidencia en el aspecto de mitigación que el Estado colombiano ha comenzado a promover algunos procesos de articulación en los diferentes organismos de la gestión pública entre los que cabe mencionar: el Fondo de Adaptación, Colombia Humanitaria, Unidad Nacional para la Atención del Riesgo, que cuentan con el apoyo de entidades regionales como la Cruz Roja, el Comité Local de prevención y Atención de Desastres (CLOPAD) y el Comité Regional de Prevención y Atención de Desastres (CREPAD).

Sin embargo, el tipo de asistencia a las comunidades vulnerables se da en un proceso asistencialista con la entrega de mercados, frazadas, alimentos y artículos de primera necesidad, tal como lo muestra el siguiente testimonio: "vino la defensa civil en un tiempo $\mathrm{y}$ les dio a las personas que estaban acampadas en el cementerio, a los que no tuvieron cómo hacer su cambuche en su casa, vino la defensa civil, les donó un cambuche grande muy bonito, con sus respectivos útiles que llevan por dentro: colchones, sabanas, ollas, cuchillo, martillos y otras cosas (Informante clave: Comunidad San Miguel, 2013). 
En cuanto al desempeño gubernamental colombiano en el tópico de la mitigación en la Costa Caribe, se nota un accionar enfocado principalmente en lo atencional de las crisis ambientales y en menor grado preventivo de las mismas dificultades climáticas, aunado a una incipiente articulación misional entre los gremios ambientales.

Una eficiente coordinación sistemática y oportuna exige las corresponsabilidades de todos los miembros de los entes públicos, es decir, la clase dirigente, empresarios, corporaciones ambientales y comunidades afectadas, para definir estrategias concertadas basadas en el conocimiento social de la variabilidad climática y el reconocimiento de la fragilidad de la geografía caribeña a las variaciones atmosféricas y la falta de previsión frente a los problemas de la región. Esto se colige en los testimonios de funcionarios de las CARs así:

He visto en otros países desarrollados, como en Holanda, los ríos constantemente los están dragando para evitar precisamente que se desborden, que se generen catástrofes y tragedias, son países que viven con el agua hasta el cuello prácticamente constantemente; un país como el de nosotros, que tenemos mucho más terreno, más cómo minimizar este impacto, no se dragan lo ríos, no se hace nada de este proceso, y luego, cuando vienen las inundaciones es que viene hacer las actuaciones, que se vienen a ver los planes de contingencia, es que vamos a ver qué vamos hacer. (Informante clave: Funcionario, 2013)

Se evidencia un gran entusiasmo en las herramientas jurídicas ambientales para enfrentar el cambio climático y sus consecuencias, tal como lo revela el siguiente testimonio:

Bueno, a raíz de la ley 1523 del 2012, que es una herramienta jurídica para implementar los planes de gestión del riesgo municipal y departamental en cuanto a la región Atlántica o Caribe y el departamento del Magdalena, encontramos nosotros que se está actuando, que se está apoyando esta ley para dinamizar la gestión del riesgo en el territorio y además crear política o proyectos que estén encaminados a la adaptación al cambio climático; hoy podemos decir que la Costa Atlántica es líder en virtud de los planes departamentales de gestión de riesgo, hoy están activados, están coordinados y articulados para prever lo que se pueda presentar en este territorio la costa norte. (Informante clave Corporación Autónoma Regional del Magdalena, 2013)

Igualmente, se expresa la necesidad de realizar algunos cambios en la ejecución de políticas estatales: "Creo que sí hay varias instituciones que pueden gestionar pero hace falta más compromiso y menos politiquería que permita mirar con objetividad las consecuencias de no actuar a tiempo sobre la influencia del cambio climático en la Sierra Nevada" (Grupo focal institucional Palmor de Varela, Magdalena, 2013). 
Como se puede observar en los testimonios anteriores, en la región Costa Atlántica se están desarrollando diversas acciones para mitigar el cambio climático; existe mayor compromiso de las autoridades ambientales frente al fenómeno climático y acciones anticipadas, oportunas, y de manera específica atienden la gestión del riesgo, aunque también se resalta que existe improvisación en los procesos e interferencias políticas que ralentizan la ejecución de los proyectos de mitigación.

En relación con la efectividad de las acciones de mitigación emprendidas en la Región Costa Atlántica, las encuestas arrojan la siguiente información:

Tabla No. 1. Efectividad de las acciones de mitigación.

\begin{tabular}{|l|c|c|}
\hline \multicolumn{1}{|c|}{ Válidos } & Frecuencia & Porcentaje \\
\hline Altamente efectivas & 2 & 0,7 \\
\hline Medianamente efectivas & 127 & 45,7 \\
\hline Poco efectivas & 149 & 53,6 \\
\hline Total encuestas & 278 & 100,0 \\
\hline
\end{tabular}

Fuente: Elaboración propia del Grupo Investigación CIMAD, región Costa Atlántica.

\section{Gráfica No.2. Efectividad de las acciones de mitigación al cambio climático.}

altamente efectivas medianamente efectivas Poco efectivas

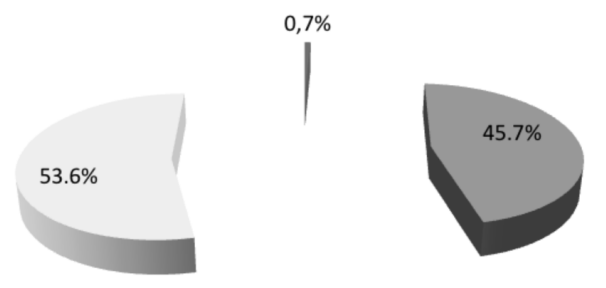

Fuente: Elaboración propia del Grupo Investigación CIMAD, región Costa Atlántica.

Sobre la efectividad de las acciones promovidas en las diferentes localidades de la Costa Atlántica para la mitigación del cambio climático, se obtuvieron los siguientes resultados: altamente efectivas: $0,7 \%$, medianamente efectivas $45,7 \%$ y poco efectivas: $53,6 \%$. Lo anterior demuestra que, en su gran mayoría, estas acciones han sido poco o medianamente efectivas. Se cree que estos resultados obtenidos se deben a los siguientes aspectos:

- La falta de voluntad política del Estado y de los entes que rigen la educación para asumir la Educación Ambiental como una necesidad sentida de la vida ciudadana, tanto para la educación formal como para la no formal aunque los avances en este aspecto se reconocen como las más importantes en mitigación. 
- El reducido número de docentes de áreas diferentes a las ciencias naturales capacitados en el campo ambiental.

- La rigidez de los esquemas pedagógicos adoptados actualmente.

- La carencia de apoyo e incentivos para el trabajo interdisciplinario en la docencia y la investigación.

La construcción de una cultura ambiental en la comunidad educativa debe traducirse en una nueva forma de vida y comportamiento de los miembros de la comunidad en relación con su entorno y con ellos mismos. El principio ético rector de este cambio debe ser el respeto al otro, a los otros y al devenir futuro.

La planificación estatal relacionada con el manejo del cambio climático en el componente mitigación se encuentra orientada a tratar los efectos y no las causas. Actualmente se vienen realizando algunas acciones atencionales preventivas como el establecimiento de bosques de sumideros de carbono y zonas de amortiguación para los excesos hídricos. Se deja a un lado un aspecto esencial e importante, la exclusión social en la que viven muchos poblados que no ven otra fuente de sustento que la extracción de los recursos naturales, constituyéndose en presión antrópica sobre los recursos ecosistémicos, lo cual tiende a acentuar la incidencia del fenómeno climatérico. Es necesario articular políticas sociales estructurales que estén encaminadas a sacar de la pobreza a estas comunidades que viven en la marginalidad económica y se ven obligados a vivir en zonas geográficas de alta vulnerabilidad a eventos climáticos.

Al mismo tiempo, se debe tener en cuenta el conocimiento social que han adquirido estas poblaciones ante las continuas exposiciones a estos sucesos atmosféricos, es decir, que se valide su participación en la toma de decisiones, proyectos y planes que tiendan a beneficiar las comunidades vulnerables.

El reconocimiento de las potencialidades que existen entre los moradores de los diferentes grupos poblacionales hace pensar en un comienzo de articulación eficiente de la política estatal para enfrentar las incertidumbres del fenómeno de la variabilidad climática y las necesidades de la población afectada.

\section{Logros en materia de mitigación y proyecciones futuras}

En este tema, los funcionarios entrevistados expresan que han sido pocos los logros en materia de mitigación, aunque existen proyecciones futuras a través de planes y proyectos, lo que dejan entrever en sus apreciaciones de esta manera:

La región digamos que logros importantes de mitigación de cambio climático, pues de verdad no ha tenido, no se han vistos esos logros en tal sentido, lo que decía, estamos en proyección, se están elaborando los planes, los proyectos a disminuir esta situación o a 
mitigar los efectos del cambio climático en la zona, digamos que lo único que se ha visto por acá es lo que viene haciendo el gobierno nacional a través del fondo de adaptación para mitigar en parte la situación de la ola invernal. (Bolívar, 2013)

En cuanto a este aspecto, otra apreciación reconoce la proyección en acciones específicas por sectores y focalizada hacia poblaciones vulnerables determinadas:

Bueno, este tema que apenas es insistido en que concierne al trabajo que nos entrega el Estado, más bien te puedo hablar de lo que se proyecta hacer, se van a hacer unas diez (10) barreras de contención sobre diez (10) puertos de agua en los que se incluyen el río Guatapuri, el río Badillo, las quebradas que están cerca de Fundación; ahí va a aportar la corporación a los bomberos, al cuerpo de bomberos, una serie de equipos para todo el tema de incendios forestales. Se está trabajando esa posibilidad para que cada sector se encargue de capacitar a poblaciones rurales por el actual mal uso que hacen con los residuos que muchas veces ocasionan los incendios forestales. (Director Corporación Autónoma del Cesar, 2013)

Otro entrevistado manifiesta la proyección con conciencia de acciones de mitigación que se asumen sectorialmente:

Hay reconocimiento en los diferentes sectores (industrial, transporte, agrícola, de residuos) con la adopción de medidas de mitigación del cambio climático; en ello la implementación de diferentes planes y programas en la reducción de las emisiones es y será de vital relevancia si se actúa con responsabilidad y conciencia en la conservación y preservación del medio ambiente. (Informante clave, 2013)

Sin embargo, es muy importante señalar que una de las proyecciones más importantes en materia de mitigación al Cambio Climático es, sin duda alguna, un proceso de formación y capacitación de diversos sectores de la población caribeña en estos asuntos, que contribuya a la construcción de una Cultura de la Prevención del Caribe Colombiano.

Los gobernantes de turno se han comprometidos a involucrar en sus planes de desarrollo y de ordenamiento territorial el componente de la gestión del riesgo, tema de vital interés que demanda una gran responsabilidad por parte del sector gubernamental. Igual el sector privado también ha empezado a adoptar programas que son pertinentes ante la crisis ambiental. Han entendido que el agravamiento de los fenómenos naturales, el modelo económico capitalista explotador y la falta de conciencia de la comunidad en general, demandan trabajo en conjunto para plantear modelos alternativos de gestión ambiental y de desarrollo que sean más sostenibles social, ambiental y culturalmente. Por ello se percibe una proyección positiva, se cuenta con nuevo ordenamiento jurídico 
con políticas públicas para la conservación de la biodiversidad que obligan a las empresas que han ocasionado daños irreversibles a los ecosistemas, especialmente aquellas relacionadas con actividades extractivas (Carbón, Níquel, Oro, Petróleo), a amortizar los pasivos ambientales y la deuda ecológica que tienen con el caribe colombiano.

En el horizonte de esta problemática, se vislumbra la necesidad de generar conciencia ambiental y social en las comunidades como un elemento estratégico en la toma de decisiones para cualquier política asociada con el cambio climático en la región.

En cuanto a proyecciones futuras referentes a subsanar esta realidad, se habla principalmente de obras de ingeniería civil como jarillones, dragados de fuentes hídricas y, en menor escala, de reforestación de bosques. En todos los departamentos de la región se está incluyendo el plan departamental para la gestión del riesgo, que genera mitigación del riesgo en todos sus componentes: riesgos para las inundaciones, deslizamiento, incendios forestales.

El manejo de la realidad del cambio climático en la región se está haciendo desde una visión antropocentrista, subvalorando la dimensión ecosistémica y las posibilidades conjuntas en su importancia para alcanzar un equilibrio sostenible desde una prospectiva egocéntrica.

En los departamentos de la región se vienen realizando planes departamentales y municipales de gestión del riesgo que buscan reducir los riesgos frente al cambio climático; no obstante, las decisiones y acciones en este tema son lentas y requieren recursos en todos los niveles territoriales. Así lo evidencian testimonios como el siguiente. "Aspiramos gestionar los recursos propios, pertinentes para poder avanzar con mayor celeridad y oportunidad el tema de la mitigación de los riesgos en el departamento del cesar." (Informante clave: Director Departamental de Gestión del Riesgo, 2013).

\section{Conclusiones}

$\mathrm{Al}$ analizar e interpretar la información recolectada en los diferentes instrumentos aplicados en el presente estudio, tales como encuestas, entrevistas semiestructuradas y grupos focales sobre las acciones de mitigación en la Costa Atlántica, la percepción generalizada es que, si bien existen políticas definidas sobre el manejo climático, apenas se está empezando a promover una articulación desde el gobierno central a través del Ministerio de Medio Ambiente y Desarrollo Sostenible, el Fondo Nacional de Adaptación, las CARs, la Unidad Nacional de Gestión del Riesgo y los comités locales de atención y prevención de desastres, para realizar acciones mitigantes en las poblaciones vulnerables, aunque estas son poco efectivas debido a la magnitud de los efectos del cambio climático.

Es necesaria la cooperación entre los entes gubernamentales, territoriales y la comunidad local para que se dé una apropiación social del conocimiento sobre el fenómeno climático y las potencialidades de las comunidades. Esto ayudaría a la definición y el 
acompañamiento de los procesos de adaptación y/o mitigación frente a la variabilidad climática, involucrando procesos de desarrollo sostenible para no generar costos de mala adaptación o sobre adaptación provenientes de medidas políticas basadas en soluciones temporales.

Los costos de las decisiones políticas desacertadas frente al cambio climático son muy superiores al de prevenir o adaptarse al mismo de manera sostenible.

Las políticas como tal existen articuladas en el Plan de Ordenamiento Territorial pero sólo como un requisito nominal hasta la aparición de la ola invernal y los eventos ante los que los entes territoriales se ven abocados a fortalecer las políticas, herramientas, mecanismos e instrumentos de gestión integral del riesgo climático para mitigar y adaptarse a tragedias presentes y futuras, signadas por la improvisación y la inmediatez.

Las acciones de mitigación desarrolladas desde el nivel estatal y privado están encaminadas principalmente a la educación ambiental y la reforestación, siendo éstas medidas viables para alcanzar una adecuada re significación cultural en la relación entre la comunidad vulnerable y su entorno natural: con la siembra de maderables para convertirlos en sumideros de carbono se buscan beneficios tributarios e incentivos económicos de los proyectos de Mecanismo de Desarrollo Limpio.

Otra importante acción mitigante que se realiza en la región está relacionada con la producción y el uso de energía alternativa eólica en menor escala en el Departamento de la Guajira. Este proyecto es posible debido a la ubicación geográfica de este departamento y a sus características fisiográficas, aunque su incidencia no es muy notable en la región Caribe por la poca inversión y participación estatal que busque la replicación de este tipo de proyectos energéticos alternativos que contribuyen a mitigar los efectos del cambio climático.

Conocer los impactos del Cambio Climático en las poblaciones vulnerables genera apropiación social del conocimiento del fenómeno climático y las potencialidades para realizar acciones mitigantes por parte de las comunidades afectadas.

Afrontar los retos del cambio climático implica incidir en los diferentes sectores gubernamentales de la sociedad civil y la población en general para que, a futuro, se fortalezcan las capacidades individuales, organizacionales e institucionales y se contribuya a cambiar los comportamientos, actitudes, prácticas y formas de organización; es prioritario que desde ahora se facilite la adopción de medidas colectivas frente al cambio climático.

Se resalta como estrategia de mitigación la generación de conocimiento social; cuando las comunidades y autoridades gubernamentales conocen del riesgo, amenazas, y los tipos de riesgos a los que están expuestos, van tomando conciencia sobre las acciones de mitigación que deben emprender para afrontar el cambio climático.

Como recomendación, se determina que la principal acción de mitigación es la generación de conocimientos mediante la cual los entes gubernamentales conozcan 
cuáles son los riesgos que se pueden presentar en su jurisdicción y los difundan en las comunidades para que éstos no afecten totalmente la población y las actividades de nuestros sectores socioeconómicos.

Hay que generar o desarrollar planes de acciones de mitigación en los que las estrategias involucren el conocimiento, la reducción del riesgo y el manejo de desastres; si se desarrolla una planificación acerca de las acciones a tomar para afrontar cada uno de estos procesos, podemos estar preparados para enfrentar el cambio climático.

Se recomienda ajustar los planes de desarrollo nacional y local para la reubicación de comunidades que por su marginalidad económica y social están expuestas a inminentes riesgos ocasionados por los efectos del cambio climático.

\section{Referencias Bibliográficas}

Departamento Nacional de Planeación. Plan Nacional de Desarrollo 2010- 2014.

Instituto de Hidrología, Meteorología y Estudios Ambientales de Colombia (2001a). Primera Comunicación Nacional ante la Conferencia Marco de las Naciones Unidas Sobre Cambio climático. Bogotá.

Instituto de Hidrología, Meteorología y Estudios Ambientales de Colombia (2001b). Primera Comunicación Nacional ante la Convención Marco de la Naciones Unidas sobre el Cambio Climático. Bogotá: Trade Link.

Instituto de Hidrología, Meteorología y Estudios Ambientales de Colombia (2001c). Primera Comunicación Nacional ante la Convención Marco de las Naciones Unidas sobre el Cambio Climático, Inventario Nacional de fuentes y sumideros de Gases de Efecto Invernadero - GEI 1990 y 1994. Bogotá: Trade Link Ltda.

Instituto de Hidrología, Meteorología y Estudios Ambientales de Colombia (2007).

Información Técnica sobre Gases de Efecto Invernadero y el Cambio Climático. METEO/Oo8.

Instituto de Hidrología, Meteorología y Estudios Ambientales de Colombia (2010). Segunda comunicación Nacional ante la Convención Marco de las Naciones Unidas sobre el Cambio Climático. Bogotá: Scripto Ltda.

Ministerio del Medio Ambiente (2000). Estudio de Estrategia Nacional para la Implementación del Mecanismo o de Desarrollo Limpio. Bogotá.

Ministerio del Medio Ambiente (2002). Lineamientos de Política de Cambio Climático. Bogotá.

Ministerio del Medio Ambiente-Instituto de Hidrología, Meteorología y Estudios Ambientales- Programa de las Naciones Unidas para el Desarrollo (2001). Primera 
Comunicación Nacional ante la Convención Marco de las Naciones Unidas sobre el Cambio Climático. Bogotá.

Ministerio de Minas y Energía (2013). Documento CONPES 3242.

Programa de las Naciones Unidas para el Desarrollo. Colombia (2010). Educación para enfrentar el cambio-. Segunda comunicación nacional ante la CMNUCC. Bogotá:

República de Colombia, Ministerio del Medio Ambiente (2000). Estudio de Estrategia Nacional para la Implementación del Mecanismo o de Des arrollo Limpio. Bogotá.

República de Colombia, Ministerio del Medio Ambiente (2002). Lineamientos de Política de Cambio Climático. Bogotá.

República de Colombia, Ministerio del Medio Ambiente y Desarrollo Sostenible. Instituto de Hidrología, Meteorología y Estudios Ambientales de Colombia (2007). Información Técnica sobre Gases de Efecto Invernadero y el Cambio Climático. METEO/oo8

República de Colombia, Ministerio del Medio Ambiente (2010). Segunda comunicación Nacional ante la Convención Marco de las Naciones Unidas sobre el Cambio Climático. Scripto Ltda., Bogotá D.C.

República de Colombia. Departamento Nacional de Planeación. Plan Nacional de Desarrollo, 2010- 2014.

República de Colombia, Consejo Nacional de Política Económica y Social, Departamento Nacional de Planeación (2010). Segunda comunicación Nacional ante la Convención Marco de las Naciones Unidas sobre el Cambio Climático. Estrategia Nacional para la articulación de políticas a nivel nacional para el Cambio climático. Bogotá: Script Ltda.

Nodos Regionales. (s.f.). Disponible en: http://redcolombianafa.org/es/nodos-regionales/ nodo-caribe 